Supporting information

\title{
Immune Responses and Anti-tumor Effect through Delivering to Antigen Presenting Cells by Optimized Conjugates Consisting of CpG- DNA and Antigenic Peptide
}

Hitomi Irie ${ }^{\dagger}, K_{0 j i}$ Morita $^{\ddagger}$, Makoto Koizumi ${ }^{\ddagger}$, Shinichi Mochizuki ${ }^{\dagger},{ }^{*}$

†Department of Chemistry and Biochemistry, The University of Kitakyushu, 1-1, Hibikino, Wakamatsu-ku, Kitakyushu, Fukuoka, 808-0135, Japan.

\#odality Research Laboratories, Daiichi Sankyo Co., Ltd., 1-2-58, Hiromachi, Shinagawa-ku, Tokyo, 140-8710, Japan.

*Corresponding author. Department of Chemistry and Biochemistry, The University of Kitakyushu, 1-1, Hibikino, Wakamatsu-ku, Kitakyushu, Fukuoka, 808-0135, Japan.

Tel: +81-93-695-3203; Fax: +81-93-695-3390

E-mail address: mochizuki@kitakyu-u.ac.jp 


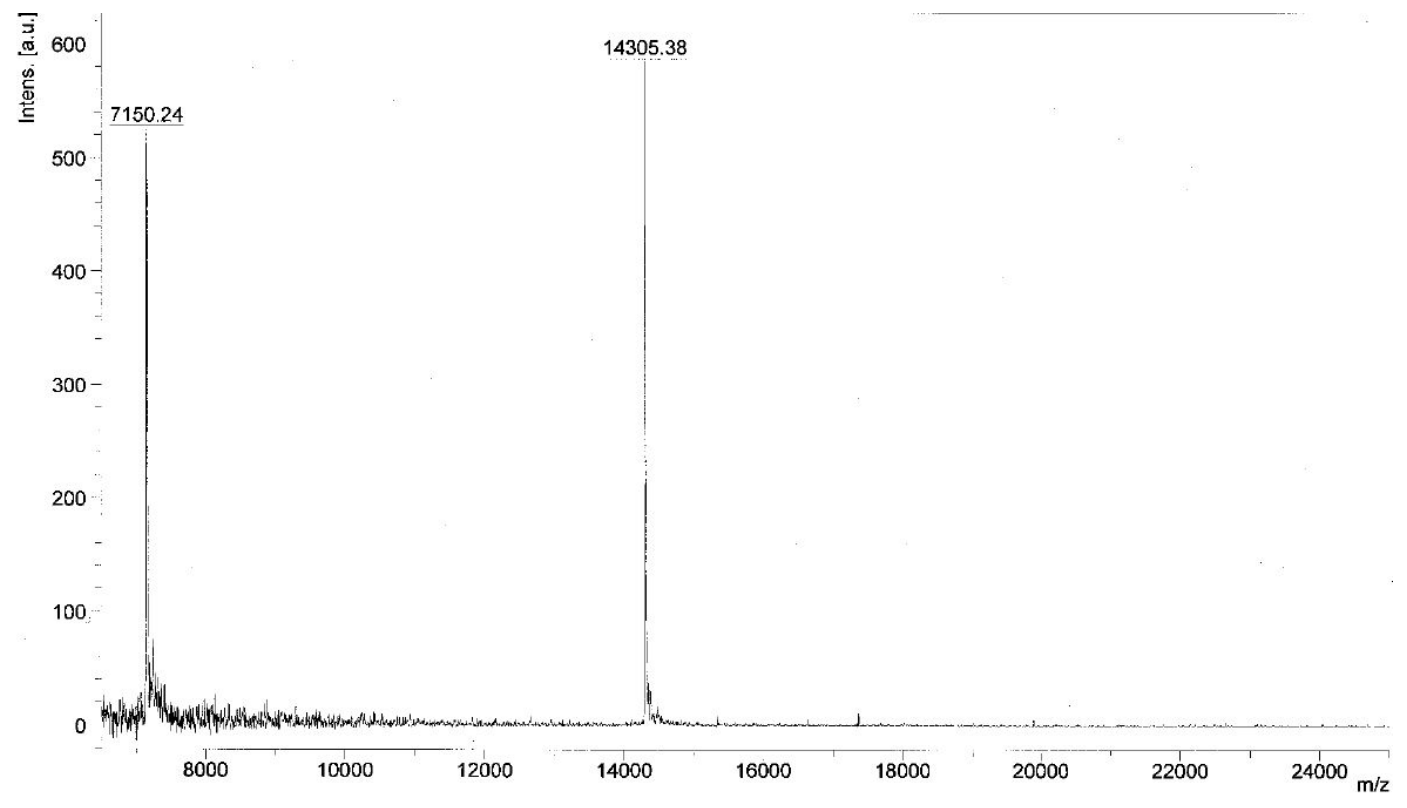

Figure S1. TOF MS spectrum of CpG40-C-OVA $257-264$. 
(A)

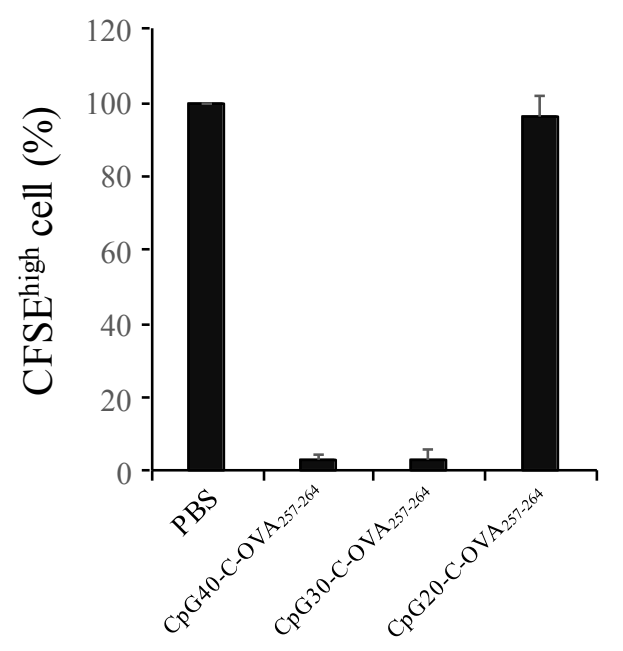

(C)

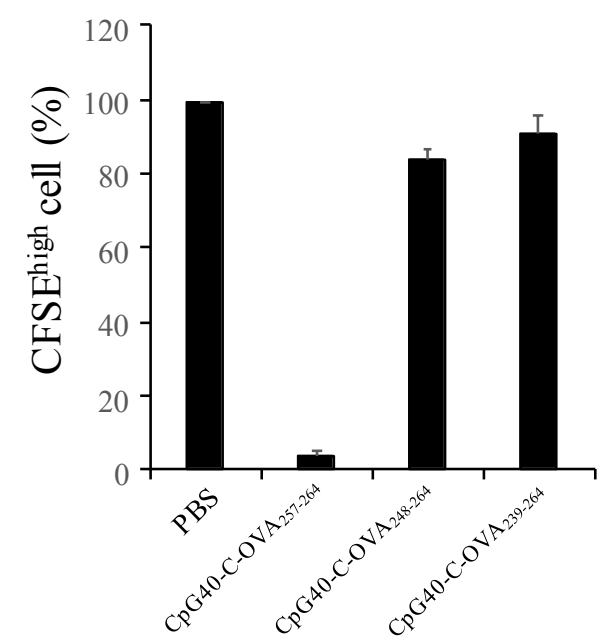

(B)

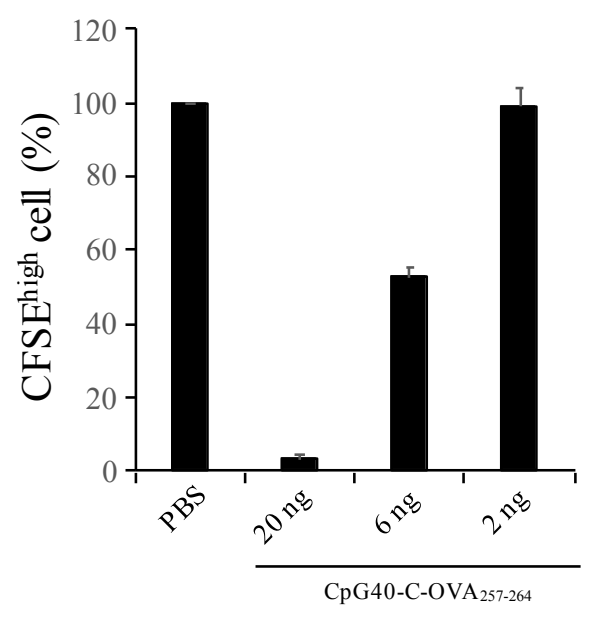

Figure S2. In vivo CTL activity for cells pulsed with $\mathrm{OVA}_{257-264}$ peptide after immunization with the indicated conjugate $\left(20 \mathrm{ng} / \mathrm{head}\right.$ as a dose of $\mathrm{OVA}_{257-264}$ peptide) (A), CpG40-C-OVA $257-264$ at the indicated doses (B), the conjugate with different lengths of peptide $(\mathrm{C})$. Results represent the mean \pm S.D. $(n=2)$. A represetative flow cytometory image is shown in Figure 3B, 3C and Figure 4, respectively. 


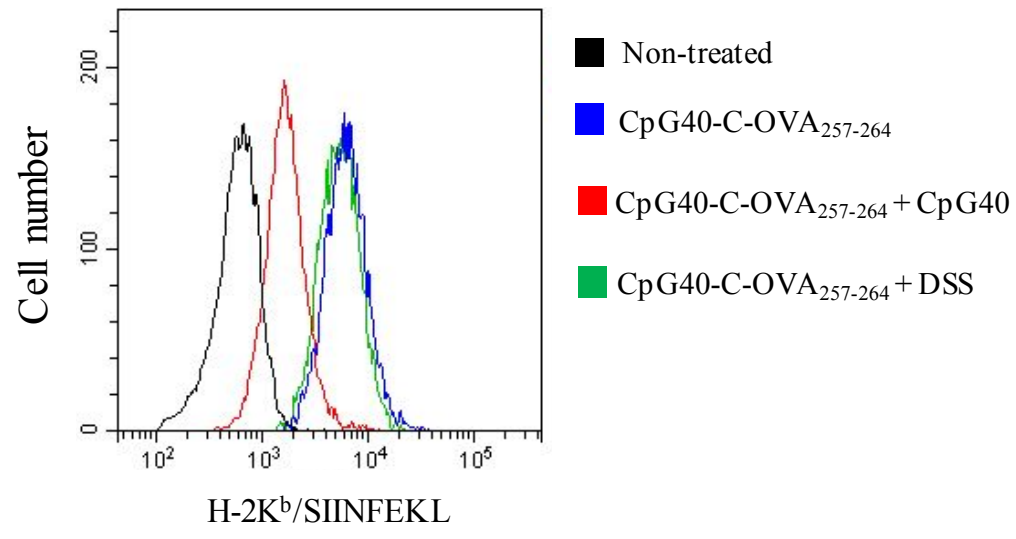

Figure S3. Antigenic peptide presentation for DC2.4 by treatment with CpG40-C$\mathrm{OVA}_{257-264}$ and excess of CpG40 or dextran sulfate (DSS). 
Table S1. List of the conjugates and the sequences

\begin{tabular}{|c|c|c|}
\hline Sample code & DNA sequence ${ }^{a}$ & Peptide sequence \\
\hline CpG20-C-OVA $257-264$ & 5'- ATCGACTCTCGAGCGTTCTC -3' & CSIINFEKL \\
\hline CpG30-C-OVA $257-264$ & 5'- GAGCGTTCTCATCGACTCTCG & CSIINFEKL \\
\hline CpG40-C-OVA $257-264$ & 5'- ATCGACTCTCGAGCGTTCTCATCGACTCTCGAGCGTTCTC -3' & CSIINFEKL \\
\hline CpG40-C-OVA $248-264$ & 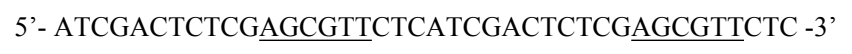 & CEVSGLEQLESIINFEKL \\
\hline CpG40-C-OVA $239-264$ & 5'- ATCGACTCTCGAGCGTTCTCATCGACTCTCGAGCGTTCTC -3' & $\begin{array}{l}\text { CMSMLVLLPDEVSGL } \\
\text { EQLESIINFEKL }\end{array}$ \\
\hline
\end{tabular}

${ }^{a}$ Underline indicates $\mathrm{CpG}$ motif. 